\title{
Comparison of the efficacy of nicardipine, a new calcium channel blocker, with nifedipine in the treatment of mild to moderate essential hypertension
}

\author{
C. Armstrong, J. Garnham and R. Blackwood \\ Wexham Park Hospital, Slough and Chiltern International Limited, UK.
}

\begin{abstract}
Summary: Thirty-nine patients with mild to moderate essential hypertension participated in a parallel, single-blind study comparing 6 weeks' treatment of nicardipine hydrochloride $(90 \mathrm{mg} /$ day $)$ with nifedipine (40 mg/day). Nicardipine-treated patients commenced therapy with a significantly higher mean supine diastolic blood pressure than the nifedipine-treated patients. There was a statistically significant fall in blood pressure (systolic and diastolic) on both treatments at the 3 and 6 week follow-up visits. On adjusting the results for the baseline inequality, no statistically significant differences were found between treatment groups.

Seven patients withdrew from nifedipine therapy and six patients withdrew from nicardipine therapy due to adverse events.

The results show that nicardipine hydrochloride at $90 \mathrm{mg} /$ day is an effective anti-hypertensive agent. The incidence and nature of adverse events were similar on the two treatments.
\end{abstract}

Introduction

The range of anti-hypertensive agents is wide. Diuretics or beta-blockers are being challenged as first-line therapy in the treatment of mild to moderate essential hypertension. Calcium channel blockers have recently been advocated as possible first-line therapy.

The main mechanism of action of calcium channel blockers in hypertensive patients is to dilate arterioles and thus decrease peripheral vascular resistance. ${ }^{1,2,3}$ Nicardipine hydrochloride is a new calcium channel blocker which is chemically related to nifedipine. It has been shown to be an effective anti-hypertensive agent ${ }^{4-8}$ both in the short and long term. Nifedipine has also been shown to be an effective anti-hypertensive agent. ${ }^{9-14}$ Both calcium channel blockers act to decrease peripheral vascular resistance thereby reducing raised blood pressure. They also have other mechanisms of action. It has been shown that nifedipine and nicardipine both have a resetting effect on the baroreflex control mechanism..$^{15,16}$ Nicardipine has also been shown to have diuretic ${ }^{16}$ and natriuretic effects, ${ }^{17}$ perhaps due to increased renal blood flow.

This study was carried out to compare the two structurally similar calcium channel blockers, nicardipine hydrochloride and nifedipine, and to determine

Correspondence: C.B. Armstrong, M.B., B.S. Wexham Park Hospital, Slough, Berks. SL2 4HL, UK.

Accepted: 27 November 1986 if they are clinically different in terms of efficacy and safety.

\section{Materials and methods}

Forty-one patients with mild to moderate essential hypertension were assessed during a treatment-free, 2 week baseline period. Two patients were withdrawn during the treatment-free period and 39 patients entered a parallel, single-blind study and were randomly allocated to receive 6 weeks' treatment with either nicardipine hydrochloride $(30 \mathrm{mg}$ capsule three times a day) or nifedipine ( $20 \mathrm{mg}$ tablet twice daily). For inclusion in the study, patients were required to have supine diastolic blood pressure (phase V) between 95 and $125 \mathrm{~mm} \mathrm{Hg}$. Patients with congestive cardiac failure, cardiac dysrhythmias, history of myocardial infarction or stroke in the 6 months prior to the study, grade 3 or 4 retinal hypertensive changes or who needed urgent reduction of blood pressure were excluded from the study, as were patients who had any serious mental or physical illness (including insulin treated diabetes mellitus).

Of the 39 patients who received study medication, 13 patients withdrew due to adverse reactions. Data for these patients are included in the efficacy analyses when available. Two patients did not take the study 
medication as instructed and two patients had diastolic blood pressures lower than $95 \mathrm{~mm} \mathrm{Hg}$ on entry to the study. These four patients were excluded from all efficacy analyses. Thus, 28 patients, 18 male and 10 female with a median age of 55 years (range 35-68), were included in the efficacy analyses. Fifteen patients received nifedipine and 13 patients received nicardipine. Two patients in the nicardipine group and one in the nifedipine group were untreated for hypertension in the 2 months prior to the study. All patients gave informed consent and approval for the study was obtained from the hospital ethical committee.

Blood pressure was measured in the right arm both supine and standing using a random zero mercury sphygmomanometer. The supine blood pressure was measured after the patient had rested for 2 minutes and the standing blood pressure was measured after the patient had been standing for 30 seconds. Weight was also recorded. Resting electrocardiograms, blood samples for haematology and biochemical analysis and urine for urinalysis were taken pre-study and at the end of the study.

Adverse events were elicited by indirect questioning at each follow-up visit and compliance was checked by a count of the returned medication. Based on the overall performance of each study medication as an effective and tolerable anti-hypertensive (global assessment), each treatment was graded as excellent, very good, good, fair or poor.

\section{Statistical analysis}

The two groups were assessed for baseline comparability of demographic variables using Wilcoxon's rank sum test and Fisher's exact test. Efficacy variables at baseline were compared using one-way analysis of variance (ANOVA). Changes from baseline blood pressure and pulse rate were analysed at each assessment using a one way ANOVA. Analysis of covariance was used to deal with the problem of baseline differences between groups in supine diastolic blood pressure.

Comparisons between baseline and follow-up values for ECG and laboratory tests were made using Wilcoxon's signed rank test (within treatment groups) and Wilcoxon's rank sum test (between treatment groups). In addition, the proportion of laboratory results increasing/decreasing from baseline, those changing from normal to abnormal and vice versa; and those with an increase or decrease from baseline of more than $50 \%$ of the normal range were all analysed using a conditional binomial test.

\section{Results}

\section{Blood pressure and heart rate}

Table I shows the reductions in systolic and diastolic blood pressure after 3 and 6 weeks of treatment. All systolic and diastolic blood pressure reductions from baseline for both treatment groups were statistically significant $(P<0.05)$. At 6 weeks the mean diastolic blood pressure was reduced by $23.1 \mathrm{~mm} \mathrm{Hg}$ for the nicardipine treatment group and $13.5 \mathrm{~mm} \mathrm{Hg}$ for the nifedipine treatment group. However, patients assigo ned to the nicardipine treatment group had statis? tically significantly higher mean supine diastolic blood pressure at baseline than patients assigned to the nifedipine treatment group. On adjusting the results for the baseline inequality no statistically significant differences were found between the two treatment groups.

Several patients withdrew from both groups during

Table I Supine and standing mean systolic and diastolic blood pressures $(\mathrm{mm} \mathrm{Hg})$

\begin{tabular}{|c|c|c|c|c|c|c|c|c|}
\hline & \multicolumn{8}{|c|}{ Change from baseline at: } \\
\hline & \multicolumn{2}{|c|}{ Baseline } & \multicolumn{2}{|c|}{ Week 3} & \multicolumn{2}{|c|}{ Week 6} & \multicolumn{2}{|c|}{ Endpoint * } \\
\hline & Nicardipine & Nifedipine & Nicardipine & Nifedipine & Nicardipine & Nifedipine & Nicardipine & Nifedipine \\
\hline \multicolumn{9}{|l|}{ Supine } \\
\hline $\begin{array}{l}\text { Systolic } \\
( \pm \text { s.d.) }\end{array}$ & $\begin{array}{l}187.4 \\
(23.9)\end{array}$ & $\begin{array}{l}183.7 \\
(24.8)\end{array}$ & -23.9 & -14.5 & -33.1 & -15.6 & -29.7 & -15.7 \\
\hline Diastolic & 113.5 & $\begin{array}{l}(24.8) \\
104.6 \dagger\end{array}$ & $\begin{array}{l}(21.8) \\
-17.8\end{array}$ & $\begin{array}{l}(19.7) \\
-8.9\end{array}$ & $\begin{array}{l}(22.7) \\
-23.1\end{array}$ & $\begin{array}{l}(23.3) \\
-13.5\end{array}$ & $\begin{array}{c}(23.6) \\
-21.2\end{array}$ & $\begin{array}{r}(21.6) \\
-11.0\end{array}$ \\
\hline ( \pm s.d.) & $(12.6)$ & $(7.6)$ & $(14.0)$ & $(10.0)$ & (14.5) & (15.5) & (14.5) & (14.9) \\
\hline \multicolumn{9}{|l|}{ Standing } \\
\hline Systolic & 164.6 & 168.7 & -19.7 & -19.6 & -25.6 & -24.0 & -24.0 & -23.4 \\
\hline ( \pm s.d.) & $(15.7)$ & $(25.0)$ & (13.6) & (26.5) & (13.0) & (23.5) & (13.4) & (22.1) \\
\hline Diastolic & 106.3 & 104.2 & -16.1 & -14.4 & -20.5 & -17.9 & -20.6 & -17.4 \\
\hline ( \pm s.d.) & $(14.8)$ & $(8.8)$ & $(17.4)$ & (15.2) & (17.7) & (13.2) & (16.2) & (12.1) \\
\hline
\end{tabular}

\footnotetext{
* Endpoint = last available value for each patient

$\dagger P<0.05$ two sample $t$ test for equality of means.
} 
active treatment. Analyses of each patient's last available blood pressure values ('end point') were performed and the results are shown in Table I.

The clinical aim in this study was to reduce supine diastolic blood pressure to between 70 and $95 \mathrm{~mm} \mathrm{Hg}$. Ten of 19 patients $(53 \%)$ in the nicardipine treatment group and 7 of 18 patients $(39 \%)$ in the nifedipine treatment group met this criterion by the end of treatment.

Supine heart rate was increased after both 3 and 6 weeks of nicardipine treatment by means of 5 and 7 beats/minute respectively. The small increase after nifedipine treatment ( 1 to 2 beats/minute) was not statistically significant. There was no statistically significant difference between treatments.

When the change in diastolic blood pressure from supine to standing was compared between treatments, no statistically significant difference was found. Four patients in the nicardipine treatment group and three patients in the nifedipine group had diastolic blood pressure decreases of greater than $15 \mathrm{~mm} \mathrm{Hg}$ on standing, but had no symptomatic complaint suggesting clinically significant postural hypotension. The difference between treatments in change from baseline was 14.0 for supine systolic blood pressure and 3.46 for adjusted diastolic blood pressure. The chances of these results being statistically significant, given the small number of patients, were $32 \%$ and $15 \%$ respectively.

\section{Other observations}

No clinically significant changes for laboratory tests were observed. The median change from baseline produced one statistically significant result which was a median increase of $0.8 \mathrm{mmol} / 1$ in urea for nicardipine-treated patients. There were no statistically significant differences between treatments for any of the laboratory tests.

At the end of the study, a mean decrease in bodyweight of $1.4 \mathrm{~kg}$ in the nicardipine treatment group and a mean increase of $0.4 \mathrm{~kg}$ in the nifedipine treatment group were seen. The difference between treatments was statistically significant $(P<0.05)$ but of doubtful clinical importance.

Resting electrocardiograms were performed both before and after treatment on 12 nicardipine-treated patients and 9 nifedipine-treated patients. There were no statistically significant changes or differences between treatments for PR and QRS intervals and QTc.

One nicardipine patient and one nifedipine patient showed new abnormalities on their electrocardiograms suggestive of worsening myocardial ischaemia. Electrocardiograms for three patients, two nicardipine-treated and one nifedipine-treated, showed improvements after 6 weeks of treatment. The two nicardipine-treated patients had less electrocar- diographic evidence of myocardial ischaemia at rest and the nifedipine-treated patient had left ventricular hypertrophy at baseline which was not detected after 6 weeks' treatment.

Thirty three of the 39 patients in this study reported adverse events. Eighteen of 21 patients receiving nicardipine and 15 of 18 patients receiving nifedipine complained of one or more adverse experiences. Six patients withdrew from nicardipine therapy and seven patients withdrew from nifedipine therapy due to adverse events which resolved on cessation of therapy. Most of the complaints were of a similar nature for both treatment groups and appeared to be due to the vasodilatory effects of the drugs, e.g. headache, oedema and flushing.

\section{Discussion}

Although nifedipine is widely used for hypertension and often successfully, there is always a need for an alternative calcium antagonist particularly as up to $20 \%$ of patients may be unable to tolerate it. Neither verapamil or diltiazem would be regarded as immediate alternatives to nifedipine. ${ }^{18}$

The main mechanism by which blood pressure is lowered by nifedipine and nicardipine is by a significant reduction in total peripheral vascular resistance. Both nifedipine and nicardipine have a predominant effect on the peripheral vasculature. Neither calcium channel blocker causes any significant electro-physiological effect on the heart. No studies have so far compared the anti-hypertensive safety and efficacy of the new calcium channel blocker, nicardipine, with the established calcium channel blocker, nifedipine.

This study has shown both drugs to reduce blood pressure significantly in patients with hypertension. The baseline inequality of the diastolic blood pressures in the two treatment groups makes it difficult to compare the absolute diastolic blood pressures. The acute response to nifedipine has been shown to be related to the pre-treatment blood pressure, ${ }^{19}$ i.e., the higher the starting blood pressure the greater the decrease in blood pressure after nifedipine treatment, but this evidence has been challenged recently by a report ${ }^{20}$ which showed that positive correlations are mathematically inevitable. When statistical correction was applied the correlation between the pre-treatment value and its change was no longer valid. In this study nicardipine patients had a significantly higher mean baseline diastolic blood pressure than the nifedipine patients and the decrease in diastolic blood pressure was larger for patients on nicardipine than nifedipine. When the diastolic blood pressure was adjusted for the baseline difference, no significant difference between treatments was found. 
This study also looked for any postural hypotension effects of the two drugs. Postural hypotension has been reported in one study with nifedipine, ${ }^{10}$ although other studies examining the effects of nicardipine ${ }^{7}$ and nifedipine ${ }^{14}$ have found no postural hypotensive effects of either drug. In this study there was no significant difference between the two drugs for change in blood pressure on standing. Patients having a significant decrease in blood pressure on standing reported no symptoms of postural hypotension.

A large proportion of patients reported one or more adverse experiences. Most of these experiences were transient and were tolerated by the patients. Seven patients in the nifedipine treatment group and six patients in the nicardipine treatment group withdrew because of adverse experiences. Twelve of the 13 patients who withdrew for adverse experiences did so within the first 3 weeks of the study. All these

\section{References}

1. Spivack, C., Ocken, S. \& Frishman, W.H. Calcium antagonists. Clinical use in the treatment of systemic hypertension. Drugs 1983, 25: 154.

2. Editorial. Calcium antagonists and blood pressure. Lancet 1983, ii: 22.

3. Hulthen, U.L., Bolli, P. \& Buhler, F.R. Calcium influx blockers in the treatment of essential hypertension. Acta Med Scand 1984, 681 (suppl): 101.

4. Asplund, J. Nicardipine hydrochloride in essential hypertension - A controlled study.

5. Forette, F., Bellet, M., Henry, J.F. et al. Effect of nicardipine in elderly hypertensive patients. J Hypertens 1984 , 2: 437.

6. Taylor, S.H., Frais, M.A. Experience with nicardipine in hypertension.

7. Jones, R.I., Hornung, R.S., Sonecha, T. \& Raftery, E.B. The effect of a new calcium channel blocker nicardipine on 24-hour ambulatory blood pressure and the pressor response to isometric and dynamic exercise. $J$ Hypertens 1983, 1: 85 .

8. Taylor, S.H., Silke, B., Ahuya, R.C. \& Okoli, R. Influence of nicardipine on the blood pressure at rest and on the pressor response to cold, isometric exertion, and dynamic exercise in hypertensive patients. $J$ Cardiovasc Pharmacol 1982, 4: 803.

9. Hallin, L., Andren, L. \& Hansson, L. Controlled trial of nifedipine and bendroflumethiazide in hypertension. Cardiovasc Pharmacol 1983, 5: 1083.

10. Murphy, M.B., Scriven, A.J.I. \& Dollery, C.T. Role of nifedipine in treatment of hypertension. Br Med J 1983, 287: 257.

11. Brennan, F., Flanagan, M., Blake, S. \& Cannon, $\mathbf{P}$. Nifedipine in the treatment of hypertension. Eur $J$ Clin Pharmacol 1983, 25: 713. symptoms resolved on cessation of treatment. The majority of the symptoms reported appeared to be due to the vasodilator properties of these drugs, e.g. flushing, headache and oedema. Two patients taking nicardipine and three taking nifedipine reported oedema. However, on examining weight changes, there was only a marginal increase in weight for patients receiving nifedipine and a decrease in weight for patients receiving nicardipine. Oedema is probably due to fluid redistribution following local vasodilatation. The small mean weight decrease seen in nicardipine-treated patients may be due to its mild diuretic effect. $^{21}$

This study has shown that nicardipine can be used successfully as monotherapy in the treatment of mild to moderate hypertension. Both nicardipine and nifedipine were shown to be safe and effective antihypertensive agents.

12. Bursztyn, M., Grossmann, E. \& Rosenthal, T. Long acting nifedipine in the treatment of severe hypertension. Eur J Clin Pharmacol 1984, 27 : 13.

13. Midtbo, K., Hals, O. \& van der Meer, J. Verapamil compared with nifedipine in the treatment of essentiah hypertension. J Cardiovasc Pharmacol 1982, 4: S363. 응

14. Hornung, R.S., Gould, B.A., Jones, R.I., Sonecha, T. Raftery, E.B. Nifedipine tablets for hypertension: study using continuous ambulatory intra-arterial recording. Postgrad Med J 1983, 59: 95.

15. Littler, W.A., Stallard, T.J., Watson, R.D.S. \& McLeay, R.A.B. The effect of nifedipine on arterial pressure and reflex cardiac control. Postgrad Med $J$ 1983, 59 (Suppl. 2): 109.

16. Young, M.A., Watson, R.D.S. \& Littler, W.A. Baroreflex setting and sensitivity after acute and chronic nifedipine therapy. Clin Sci 1984, 66: 233.

17. Van Schaik, B.A.M., Van Nistelrooy, A.E.Y. \& Geyskes, G.G. Antihypertensive and renal effects of nicardipine. Br J Clin Pharmacol 1984, 18: 57.

18. Henry, P.D. Calcium ions (Ca) antagonists: Mechanisms of action and clinical applications. Pract Cardiol 1979, 5: 145-156.

19. MacGregor, G.A., Markandu, N.D., Rotellar, C., Smith, S.J. \& Sagnella, G.A. The acute response to nifedipine is related to pre-treatment blood pressure. Postgrad Med J 1983, 59 (Suppl. 2): 91.

20. Gill, J.S., Zezulka, A.V., Beevers, D.G. \& Davies, P. Relation between initial blood pressure and its fall with treatment. Lancet 1985, i: 567.

21. Young, M.A., Watson, R.D.S., Stallard, T.J. \& Littler, W.A. Calcium channel blockers - useful diuretics?. Clin Sci 1984, 66: 38P. 